\title{
Pharmacokinetic Investigation of Main Bioactive Components from Epimedium-Derived Flavonoids in Rabbit Serum by Ultra-Performance Liquid Chromatography Tandem Mass Spectrometry
}

\author{
Liufang Hu ${ }^{1,2}$, Yuanru Zheng ${ }^{1,2}$, Yi Zhang ${ }^{1,2}$, Zifei Qin ${ }^{1,2,3^{*}}$, Yi Dai ${ }^{1,2}$, Lin Qin ${ }^{4}$, Zhihong Yao ${ }^{1,2^{*}}$ and Xinsheng Yao ${ }^{1,2,3}$ \\ ${ }^{1}$ College of Pharmacy, Jinan University, Guangzhou 510632, China \\ ${ }^{2}$ Guangdong Provincial Key Laboratory of Pharmacodynamic Constituents of TCM and New Drugs Research, College of Pharmacy, Jinan University, Guangzhou 510632, \\ PR China \\ ${ }^{3}$ Integrated Chinese and Western Medicine Postdoctoral Research Station, Jinan University, Guangzhou 510632, PR China \\ ${ }^{4}$ Musculoskeletal Research Laboratory, Department of Orthopedics and Traumatology, The Chinese University of Hong Kong, Satin, NT Hong Kong SAR, PR China
}

\begin{abstract}
Background: Total Epimedium-derived flavonoids (EF) were reported to be a group of major bioactive constituents present in genus Epimedium for the treatment of osteonecrosis. The present study aimed to investigate the pharmacokinetics of main bioactive components of EF in rabbit.

Methods: A rapid and sensitive method for the simultaneous determination of icariin, icariside I, icariside II and icaritin in serum was established and validated by ultra-performance liquid chromatography coupled with quadrupole time-of-flight tandem mass spectrometry (UPLC-Q-TOF/MS). Serum samples were pretreated with water-saturated ethyl acetate. Furthermore, sample separation was achieved on an Waters BEH C18 column $(50 \mathrm{~mm} \times 2.1 \mathrm{~mm}, 1.7 \mu \mathrm{m})$ by gradient elution with acetonitrile-water (both including $0.1 \%$ formic acid) as mobile phase. Finally, pharmacokinetics of EF in rabbit serum was investigated.
\end{abstract}

Results: All calibration curves displayed excellent linearity $\left(r^{2}>0.99\right)$ within $1.2 \sim 24 \mathrm{ng} / \mathrm{mL}$. The assay was specific, precise and accurate, as demonstrated with intra-run precisions not more than $12.6 \%$, inter-run precisions less than $9.9 \%$ and accuracies between $-4.4 \%$ and $9.3 \%$. Meanwhile, the matrix effects, extraction recoveries and stabilities were all satisfactory. Moreover, this validated method was successfully applied to analyse the pharmacokinetic of EF in rabbit serum after oral dosage of $80 \mathrm{mg} / \mathrm{kg}$.

Conclusion: These results would enlarge our knowledge about main bioactive components of EF for the treatment of osteonecrosis in rabbit.

Keywords: Epimedium-derived flavonoids; Osteonecrosis; Main bioactive components; Pharmacokinetic; Rabbit; UPLC-Q-TOF/MS

\section{Introduction}

Herba Epimedii, the dried aerial parts of Epimedium L. (Berberidaceae), are a widely used Chinese medicine for impotence and bone loss in East Asian countries for thousands of years [1-3]. Epimedium-derived flavonoids (EF) was reported to be a group of major bioactive constituents presents in Epimedium for promoting osteoblastogenesis, preventing bone loss and steroid-associated osteonecrosis [4-6]. In clinics, a 24-month randomized double-blind placebo-controlled clinical trial had proved that the Epimediumderived phytoestrogen flavonoids could exert beneficial effects on preventing bone loss in late postmenopausal women, without resulting in a detectable hyperplasia effect on the endometrium [7]. Among these Epimedium-derived flavonoids, icariin and icaritin were considered as the potential agents for treating steroid associated osteonecrosis [8-13].

These biological activities above had stimulated increasing interest in the in vivo metabolism of EF. Poor oral bioavailability and first-pass effect of prenylflavonoids had been performed by human intestinal Caco-2 and perfused rat intestinal models [14]. Meanwhile, the amount and bioactivity of intestinal flora and enzymes changed in ovariectomized rats, which affected the intestinal absorption and hydrolysis of epimedium total flavonoids [15]. In previous study, we found that icariin, icarisid I, icarisid II and icaritin were the main absorbed components in rabbit's serum after intragastric administration of EF [16]. In rabbit's intestine, the main metabolic pathways of EF were the sequential deglycosylation metabolism in rat intestine to form secondary glycosides and final product icaritin [17]. Additionally, icaritin was easily conjugated with a glucuronic acid to form phase II metabolites in liver, which indicated that biliary clearance was one of the major routes of excretion [18]. To better understand the metabolic fate, the pharmacokinetics of EF were conducted in this study. In the past decade, ultra-performance liquid chromatography coupled with quadrupole time-of-flight mass spectrometry (UPLC/Q-TOF-MS) had been widely introduced to rapidly identify and quantify trace components in complex samples, which exhibited a lot of superiority (superior resolution, high sensitivity and sample throughout) $[19,20]$. In this study, a rapid and feasible method for icariin, icariside I, icariside II and icaritin was established and validated. Furthermore, the method was successfully applied to investigate the pharmacokinetics of the main components of EF in rabbit serum after oral administration by UPLC/Q-TOF-MS. Taken together, this study would provide a basis

*Corresponding authors: Zhihong Yao, College of Pharmacy, Jinan University, Guangzhou 510632, PR China, Tel: 862085221767; Fax: 862085221559; E-mail: tyaozh@jnu.edu.cn (or) yaozhihong.jnu@gmail.com

Zifei Qin, College of Pharmacy, Jinan University, Guangzhou 510632, PR China Tel: 862085220010; E-mail: qzf1989@163.com

Received May 02, 2017; Accepted May 08, 2017; Published May 13, 2017

Citation: Hu L, Zheng Y, Zhang Y, Qin Z, Dai Y, et al. (2017) Pharmacokinetic Investigation of Main Bioactive Components from Epimedium-Derived Flavonoids in Rabbit Serum by Ultra-Performance Liquid Chromatography Tandem Mass Spectrometry. Med Chem (Los Angeles) 7: 869-874. doi: 10.4172/2161-0444.1000444

Copyright: @ $2017 \mathrm{Hu}$ L, et al. This is an open-access article distributed under the terms of the Creative Commons Attribution License, which permits unrestricted use, distribution, and reproduction in any medium, provided the original author and source are credited. 
Citation: Hu L, Zheng Y, Zhang Y, Qin Z, Dai Y, et al. (2017) Pharmacokinetic Investigation of Main Bioactive Components from Epimedium-Derived Flavonoids in Rabbit Serum by Ultra-Performance Liquid Chromatography Tandem Mass Spectrometry. Med Chem (Los Angeles) 7: 869874. doi: 10.4172/2161-0444.1000444

of the mechanisms of action and further pharmacological studies of EF for the treatment of osteonecrosis.

\section{Materials and Methods}

\section{Materials and reagents}

Epimedium-derived flavonoid (EF) glycosides (icariin $\geqq 83 \%$, epimedoside $\mathrm{A} \leqq 1.65 \%$, hexandraside $\mathrm{F}<0.91 \%$, epimedin $\mathrm{A}<1.18 \%$, epimedin $\mathrm{B} \leqq 1.52 \%$, epimedin $\mathrm{C}<4.1 \%$ and icariside $\mathrm{II}<1.84 \%$ ) were provided by Beijing TongRenTang Health Pharmaceutical Corp., Ltd (Beijing, China). Icariin, icariside I, icariside II and icaritin (shown in Figure 1) were all purchased from Shanghai Winherb Medical Science Corp., Ltd. (Shanghai, China) with purity above 98\%. Acetonitrile and Methanol were of HPLC grade and purchased from Dikma Technologies Inc. (Beijing, China). Watsons water was purchased from Beijing Watsons Water Corp., Ltd. (Beijing, China). Other reagents were of analytical grade.

\section{Animals}

Specific pathogen free (SPF) male New Zealand rabbits $(2.75 \pm 0.25$ $\mathrm{kg}$ ) were provided by Guangdong Medical Laboratory Animal Center (Guangdong, PR China). The rabbits were kept in a designated animal room at constant temperature $(25 \pm 2)^{\circ} \mathrm{C}$ and humidity $(55 \pm 10) \%$ with $12 \mathrm{~h}$ of light/dark per day and free access to water and food. The experimental protocol was approved by the Ethics Review Committee for Animal Experimentation of Jinan University. All procedures were in accordance with the Guide for the Care and Use of Laboratory Animals (National Institutes of Health).

\section{Preparation of standard solutions}

Stock solutions of four flavonoids were prepared separately in methanol at a concentration of $36 \mu \mathrm{g} / \mathrm{mL}$. Calibration samples were prepared by spiking $100 \mu \mathrm{L}$ of the appropriate working solution to $2 \mathrm{~mL}$ of blank rabbit serum. The obtained serum concentrations were 1.2, 3.0, $6.0,9.0,12.0,18.0$ and $24.0 \mathrm{ng} / \mathrm{mL}$. The quality control samples (QCs) were independently prepared in the same manner to obtain the serum concentrations of 3.0, 9.0 and $18.0 \mathrm{ng} / \mathrm{mL}$ representing low, medium and high concentration levels, respectively. All the solutions were stored at $4^{\circ} \mathrm{C}$ before use.

\section{Serum sample preparation}

Each serum sample $(2 \mathrm{~mL})$ was transferred to a $15 \mathrm{~mL}$ polypropylene tube containing $100 \mu \mathrm{L}$ of methanol. Water-saturated ethyl acetate (4 $\mathrm{mL}$ ) was added, then vortex-mixed vigorously for $1 \mathrm{~min}$ and centrifuged at $10000 \mathrm{rpm}$ for $10 \mathrm{~min}$. The supernatant was then transferred and<smiles>[R20]Oc1cc(O)c2c(=O)c(O[R2])c(-c3ccc(OC)cc3)oc2c1CC=C(C)C</smiles>

icariin: $R_{1}=R h a, R_{2}=G l c$

icariside $\mathrm{I}: \mathrm{R}_{1}=\mathrm{H}, \mathrm{R}_{2}=\mathrm{Glc}$

icariside II: $\mathrm{R}_{1}=\mathrm{Rha}, \mathrm{R}_{2}=\mathrm{H}$

icaritin: $\mathrm{R}_{1}=\mathrm{H}, \mathrm{R}_{2}=\mathrm{H}$

Figure 1: Chemical structures of icariin, icariside I, icariside II and icaritin. evaporated to dryness under nitrogen stream at room temperature. The residue was re-dissolved in $500 \mu \mathrm{L}$ of methanol, and and $4 \mu \mathrm{L}$ aliquots were then injected into the UPLC-Q-TOF/MS system.

\section{UPLC/Q-TOF-MS conditions}

UPLC was performed using an Acquity ${ }^{\mathrm{TM}}$ UPLC system (Waters Corporation, Manchester, U.K.) with an auto-sampler at $4^{\circ} \mathrm{C}$. Chromatographic separation was performed on an Acquity UPLC BEH $\mathrm{C}_{18}$ column $(1.7 \mu \mathrm{m}, 2.1 \times 50 \mathrm{~mm}$; Serial No: 01963110215567 , Waters Corporation, USA) coupled with a VanGuard ${ }^{\mathrm{TM}}$ pre-column $(1.7 \mu \mathrm{m}$, $2.1 \times 5 \mathrm{~mm}$; Waters Corporation, USA). The mobile phase consisted of solvent A (water including $0.1 \%$ formic acid) and solvent B (acetonitrile including $0.1 \%$ formic acid) delivered at a flow rate of $0.6 \mathrm{~mL} / \mathrm{min}$. The gradient program was as follows: $0 \sim 0.3 \mathrm{~min}, 30 \% \mathrm{~B} ; 0.5 \mathrm{~min}, 40 \% \mathrm{~B} ; 2$ min, $52 \%$ B; 3 min $60 \%$ B; 3.4 min, $100 \%$ B; 3.8 min, $100 \%$ B; 3.81 min, $30 \% \mathrm{~B} ; 4 \mathrm{~min}, 30 \% \mathrm{~B}$. The column temperature was maintained at $25^{\circ} \mathrm{C}$ and the injection volume was $4 \mu \mathrm{L}$. The UPLC system was coupled to a hybrid quadrupole orthogonal time-of-flight (Q-TOF) tandem mass spectrometer (SYNAPT ${ }^{\mathrm{TM}}$ G2 HDMS, Waters, Manchester, U.K.) with electrospray ionization (ESI). The ESI source was operated in the positive ionization mode and optimized conditions for maximum detection of metabolites were as follows: capillary voltage, $3.0 \mathrm{kV}$; sampling cone, $35 \mathrm{~V}$; extraction cone, $4.0 \mathrm{~V}$; source temperature, $100^{\circ} \mathrm{C}$; desolvation temperature, $300^{\circ} \mathrm{C}$. The cone and desolvation gas $\left(\mathrm{N}_{2}\right)$ flows were set at 50 and $800(\mathrm{~L} / \mathrm{h})$. The full scan mass range was 50 1200 Da. Selective reactions monitoring (SRM) parameter of icariin, icariside I, icariside II and icaritin were as follow. The $\mathrm{m} / z 677.244 \rightarrow 369.134$ transition with a collision energy (CE) of $25 \mathrm{eV}$ for icariin form 0.50 to $0.84 \mathrm{~min} ; \mathrm{m} / \mathrm{z}$ $531.186 \rightarrow 369.134$ transition with a CE of $25 \mathrm{eV}$ for icariin form 1.10 to $1.44 \mathrm{~min} ; \mathrm{m} / z 369.134 \rightarrow 313.071$ transition with a $\mathrm{CE}$ of $30 \mathrm{eV}$ for icariin form 1.36 to $1.70 \mathrm{~min} ; \mathrm{m} / z 369.134 \rightarrow 313.071$ transition with a $\mathrm{CE}$ of $25 \mathrm{eV}$ for icariin form 3.00 to $3.50 \mathrm{~min}$. The method employed lock spray with leucine enkephalin $(\mathrm{m} / z 556.2771$ in positive ion mode and $m / z 554.2615$ in negative ion mode) to ensure mass accuracy.

\section{Method validation}

The method was validated for specificity, linearity, matrix effects, extraction recovery, precision, accuracy and stability according to the Guidance for Industry: bioanalytical method validation from the US Food Drug Administration (FDA) [21]. To ensure there were no significant endogenous interferences, specificity was determined by comparing the chromatograms obtained for each blank serum sample (from six different rabbits), rabbit serum with standard solutions at LLOQ concentrations and serum samples collected $0.5 \mathrm{~h}$ after oral administration of EF with a dose of $80 \mathrm{mg} / \mathrm{kg}$.

For the linearity, calibration curves were constructed by plotting peak area of analytes $(\mathrm{y})$ versus respective serum concentrations $(\mathrm{x})$ using a $1 / x^{2}$ weighting factor and linear least-squares regression analysis, and the slope, intercept and correlation coefficient of each curve were determined. The LLOQ was defined as the lowest concentration in the standard curve that can be measured with acceptable accuracy and precision. The accuracies and inter/intra-day precisions of the assay were evaluated by determining six replicates of QC samples (at low, middle and high concentrations) on three consecutive days.

Extraction recoveries (ER) and matrix effects (ME) were evaluated using a published experimental protocol [22]. The peak areas of three different concentrations of analytes within quality control (QC) samples (3.0, 9.0 and $18.0 \mathrm{ng} / \mathrm{mL}$ ) were defined as A1. A2 referred to the peak areas pertaining to analytes within extracted control serum samples reconstituted with standard solutions at three concentrations. 
Citation: Hu L, Zheng Y, Zhang Y, Qin Z, Dai Y, et al. (2017) Pharmacokinetic Investigation of Main Bioactive Components from Epimedium-Derived Flavonoids in Rabbit Serum by Ultra-Performance Liquid Chromatography Tandem Mass Spectrometry. Med Chem (Los Angeles) 7: 869874. doi: 10.4172/2161-0444.1000444

A3 corresponded to the responses of analytes obtained by directly injecting the corresponding pure reference standards at three QC levels. Extraction recoveries and matrix effects were calculated as follows: $\mathrm{ER} \%=\mathrm{A} 1 / \mathrm{A} 2 \times 100 \%$, and $\mathrm{ME} \%=\mathrm{A} 2 / \mathrm{A} 3 \times 100 \%$. The percentage of the relative standard deviation (RSD\%) was used to report the precision.

The stability of icariin, icariside I, icariside II and icaritin in rabbit serum were analyzing triplicate QC samples $(n=3)$ at concentrations of 3.0, 9.0, $18.0 \mathrm{ng} / \mathrm{mL}$ stored for $8 \mathrm{~h}$ at ambient temperature, for 48 $\mathrm{h}$ at $-80^{\circ} \mathrm{C}$ and also following three cycles of freezing at $-80^{\circ} \mathrm{C}$ and thawing, for $24 \mathrm{~h}$ at ambient temperature after water-saturated ethyl acetate extraction. Concentrations of these serum samples following the above storage process were compared to those of freshly prepared serum samples to verify the assay stability.

\section{Pharmacokinetic analysis}

The proposed method was applied to investigate the pharmacokinetics of EF in rabbit serum. Three New-Zealand male rabbit (SPF degree) was given to rabbit with $\mathrm{EF}$ at a dose of $80 \mathrm{mg}$ / $\mathrm{kg}$. Blood samples were collected in blood collection tubes before and $0.5,1.0,2.0,3.0,4.0,5.0,6.0,7.0$ and $8.0 \mathrm{~h}$ time points post-dosing. After collecting, rabbit was added the same amount of saline each time. The serum samples were separated by incubation for $2 \mathrm{~h}$ at $37^{\circ} \mathrm{C}$ and centrifugation at $3000 \mathrm{rpm}$ for $10 \mathrm{~min}$, which were stored at $-80^{\circ} \mathrm{C}$ until analysis.

(A)

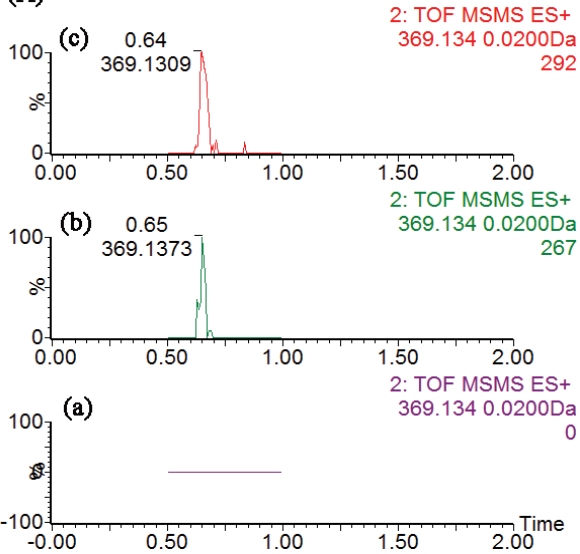

(C)

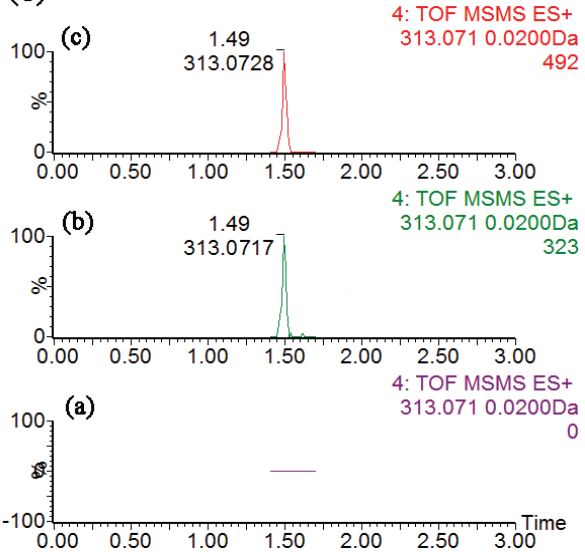

\section{Results and Discussion}

\section{Specificity}

The specificity result showed that no significant interferences from endogenous substances were observed at the retention times of icariin, icariside I, icariside II and icaritin. The selective reaction monitoring (SRM) chromatograms of icariin, icariside I, icariside II and icaritin were shown in Figure 2.

\section{Linearity of calibration curves and lower limits of quantification}

The calibration curves were linear over the concentration range of $1.2 \sim 24.0 \mathrm{ng} / \mathrm{mL}$ for icariin, icariside I, icariside II and icaritin. Correlation coefficients generated by linear regression with a $1 / x^{2}$ weighting factor ranged from 0.9914 to 0.9945 . The detailed linear correlation paramete $s$ and LODs and LOQs of four analytes in rabbit serum after oral administration of EF were displayed in Table 1 . The LLOQ was confirmed to be $1.2 \mathrm{ng} / \mathrm{mL}$, at which the intra- and inter-run accuracies were within $\pm 20 \%$. Intra- and inter-run precisions at the LLOQ level were not more than $20 \%$.

\section{Precision and accuracy}

Accuracy and precision of the assay were summarized in Table 2. Both intra- and inter-run accuracies for all tested concentrations were

(B)
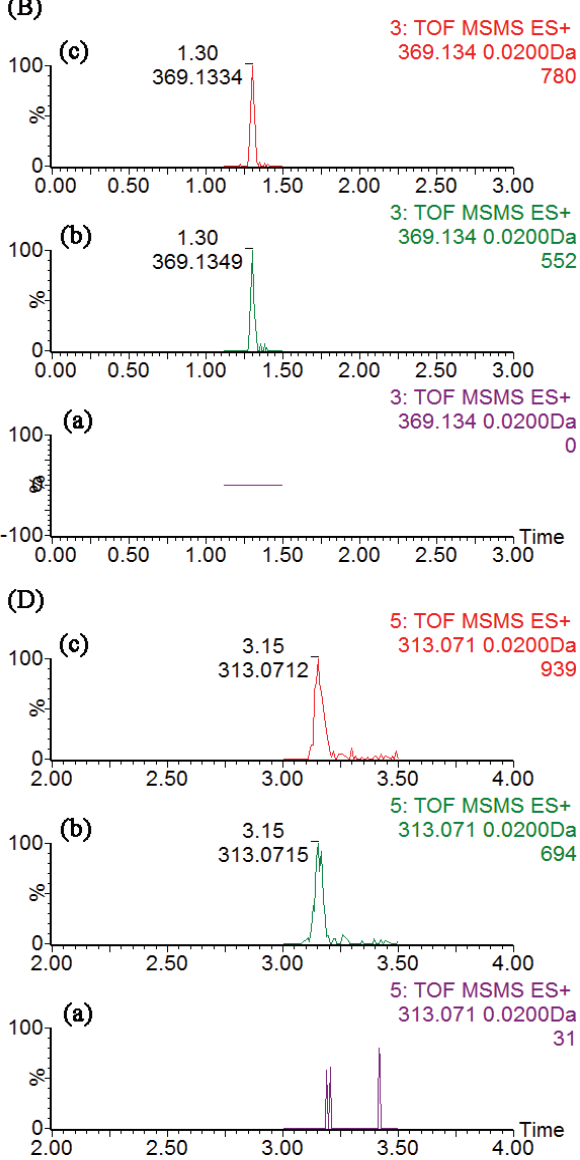

Figure 2: Selective reaction monitoring (SRM) chromatograms of icariin (A), icariside I (B), icariside II (C) and icaritin (D) in blank serum (a), drug serum at $0.5 \mathrm{~h}$ after oral administration; (b), blank serum spiked with standards resolution at LLOQs (c). 
Citation: Hu L, Zheng Y, Zhang Y, Qin Z, Dai Y, et al. (2017) Pharmacokinetic Investigation of Main Bioactive Components from Epimedium-Derived Flavonoids in Rabbit Serum by Ultra-Performance Liquid Chromatography Tandem Mass Spectrometry. Med Chem (Los Angeles) 7: 869874. doi: 10.4172/2161-0444.1000444

between $-4.4 \%$ and $9.3 \%$. Intra-run precisions were not more than $12.6 \%$ and inter-run precisions were not more than $9.9 \%$. These results demonstrated that accuracy and precision of the assay were within acceptable limits.

\section{Matrix effects and extraction recovery}

The results of the matrix effect and extraction recovery were both summarized in Table 3, which meant that no significant matrix effect for icariin, icariside I, icariside II and icaritin in rabbit serum. The matrix effect of four analytes were from $94.5 \%$ to $113.9 \%$ with RSD values less than $14.8 \%$. The extraction recovery of icariin, icariside I, icariside II and icaritin were all above $89.1 \%$ with RSD values no more than $14.4 \%$. The results demonstrated that the recoveries obtained were consistent and reproducible and did not indicate significant enhancement or suppression of ionization for any of the analytes studied.

\section{Stability}

The results of stability experiments were given in Table 4 . The data indicated that the serum samples was stable after frozen at $-80^{\circ} \mathrm{C}$ for $48 \mathrm{~h}$, after three freeze-thaw cycles, after water-saturated ethyl acetate

\begin{tabular}{|c|c|c|c|c|c|c|}
\hline Compound & Slope & Intercept & Concentration range $\mathbf{( n g} / \mathbf{m L})$ & $\boldsymbol{r}^{2}$ & LODs $\mathbf{( n g} / \mathbf{m L})$ & LOQs $(\mathbf{n g} / \mathbf{m L})$ \\
\hline icariin & 3.1998 & 0.1275 & $1.2 \sim 24.0$ & 0.9914 & 0.3 & 0.6 \\
\hline icariside I & 4.3561 & -0.9366 & $1.2 \sim 24.0$ & 0.9926 & 0.3 & 0.6 \\
\hline icariside II & 1.2632 & -0.5109 & $1.2 \sim 24.0$ & 0.9945 & 0.3 & 0.6 \\
\hline icaritin & 7.0131 & 1.6212 & $1.2 \sim 24.0$ & 0.9939 & 0.3 & 0.6 \\
\hline
\end{tabular}

Table 1: Linear correlation parameters and LLOQs of four analytes in rabbit serum after oral administration of Epimedium-derived flavonoids extracts.

\begin{tabular}{|c|c|c|c|c|c|c|c|}
\hline \multirow[t]{2}{*}{ Compounds } & \multirow[t]{2}{*}{ Conc. (ng/mL) } & \multicolumn{3}{|c|}{ Intra-run (n=5) } & \multicolumn{3}{|c|}{ Inter-run ( $n=15)$} \\
\hline & & Measured Conc. (ng/mL) & RE (\%) & RSD (\%) & Measured Conc. (ng/mL) & RE (\%) & RSD (\%) \\
\hline \multirow[t]{3}{*}{ icariin } & 3.0 & $3.1 \pm 0.32$ & 10.4 & 3.3 & $3.04 \pm 0.30$ & 9.9 & 1.3 \\
\hline & 9.0 & $9.1 \pm 0.73$ & 8.1 & 1.1 & $9.3 \pm 0.64$ & 6.9 & 3.3 \\
\hline & 18.0 & $17.2 \pm 1.37$ & 8.0 & -4.4 & $17.7 \pm 1.07$ & 6.1 & -1.7 \\
\hline \multirow[t]{3}{*}{ icariside I } & 3.0 & $3.06 \pm 0.36$ & 11.8 & 2.0 & $3.08 \pm 0.27$ & 8.8 & 2.7 \\
\hline & 9.0 & $9.08 \pm 1.14$ & 12.6 & 0.9 & $9.02 \pm 0.72$ & 8.0 & 0.2 \\
\hline & 18.0 & $17.54 \pm 0.72$ & 4.2 & -2.6 & $17.43 \pm 1.19$ & 6.9 & -3.2 \\
\hline \multirow[t]{3}{*}{ icariside II } & 3.0 & $2.88 \pm 0.33$ & 11.5 & -4.0 & $3.05 \pm 0.30$ & 9.9 & 1.7 \\
\hline & 9.0 & $8.76 \pm 0.99$ & 11.4 & -2.7 & $9.2 \pm 0.91$ & 9.9 & 2.2 \\
\hline & 18.0 & $17.54 \pm 1.00$ & 5.8 & -2.6 & $17.75 \pm 0.82$ & 4.7 & -1.4 \\
\hline \multirow[t]{3}{*}{ icaritin } & 3.0 & $3.28 \pm 0.26$ & 8.0 & 9.3 & $3.14 \pm 0.26$ & 8.3 & 4.7 \\
\hline & 9.0 & $9.22 \pm 0.63$ & 6.9 & 2.4 & $9.19 \pm 0.60$ & 6.6 & 2.1 \\
\hline & 18.0 & $18.58 \pm 1.18$ & 6.4 & 3.2 & $18.11 \pm 0.91$ & 5.1 & 0.6 \\
\hline
\end{tabular}

Table 2: Inter/intra-day accuracy and precision for icariin, icariside I, icariside II and icaritin in rabbit serum after oral administration of Epimedium-derived flavonoids extracts.

\begin{tabular}{|c|c|c|c|c|c|}
\hline Compound & Concentration $(\mathrm{ng} / \mathrm{mL})$ & ME (\%) & RSD (\%) & ER (\%) & RSD (\%) \\
\hline \multirow[t]{3}{*}{ icariin } & 3.0 & $113.2 \pm 9.7$ & 14.4 & $98.7 \pm 6.1$ & 14.3 \\
\hline & 9.0 & $101.4 \pm 6.9$ & 6.8 & $104.0 \pm 6.3$ & 6.1 \\
\hline & 18.0 & $104.7 \pm 6.1$ & 5.8 & $89.1 \pm 6.2$ & 7.9 \\
\hline \multirow[t]{3}{*}{ icariside I } & 3.0 & $112.1 \pm 11.5$ & 14.7 & $110.7 \pm 7.1$ & 10.8 \\
\hline & 9.0 & $113.9 \pm 8.4$ & 6.8 & $99.3 \pm 10.3$ & 10.4 \\
\hline & 18.0 & $111.3 \pm 7.4$ & 6.1 & $113.8 \pm 6.1$ & 5.3 \\
\hline \multirow[t]{3}{*}{ icariside II } & 3.0 & $94.5 \pm 15.9$ & 14.8 & $100.6 \pm 10.6$ & 13.5 \\
\hline & 9.0 & $108.0 \pm 10.8$ & 10.0 & $101.9 \pm 10.7$ & 11.5 \\
\hline & 18.0 & $103.0 \pm 8.9$ & 8.7 & $90.1 \pm 7.6$ & 8.4 \\
\hline \multirow[t]{3}{*}{ icaritin } & 3.0 & $106.1 \pm 9.5$ & 14.4 & $116.1 \pm 9.6$ & 14.4 \\
\hline & 9.0 & $106.7 \pm 9.9$ & 8.3 & $99.8 \pm 5.5$ & 5.5 \\
\hline & 18.0 & $108.9 \pm 10.7$ & 7.2 & $105.6 \pm 4.9$ & 9.6 \\
\hline
\end{tabular}

Table 3: Matrix effect (ME) and extraction recovery (ER) of icariin, icariside I, icariside II and icaritin in rabbit serum ( $n=5)$.

\begin{tabular}{|c|c|c|c|c|c|c|c|c|c|}
\hline \multirow[t]{2}{*}{ Compound } & \multirow[t]{2}{*}{ Conc. (ng/mL) } & \multicolumn{2}{|c|}{ At $-80^{\circ} \mathrm{C}$ for $48 \mathrm{~h}$} & \multicolumn{2}{|c|}{ Three freeze-thaw cycles } & \multicolumn{2}{|c|}{$\begin{array}{l}\text { Extracted samples at room } \\
\text { temperature for } 24 \mathrm{~h}\end{array}$} & \multicolumn{2}{|c|}{ At room temperature for $8 \mathrm{~h}$} \\
\hline & & $\mathrm{RE}(\%)$ & RSD(\%) & RE(\%) & RSD(\%) & $\mathrm{RE}(\%)$ & RSD(\%) & $\operatorname{RE}(\%)$ & RSD(\%) \\
\hline \multirow[t]{2}{*}{ icariin } & 3.0 & -4.3 & 11.2 & 10 & 6.1 & 10 & 13.4 & 1 & 8.3 \\
\hline & 18.0 & 4.4 & 9.1 & 6.7 & 0.9 & 3.3 & 0.6 & 6.8 & 1.4 \\
\hline \multirow[t]{2}{*}{ icariside I } & 3.0 & -2.3 & 8.6 & 10 & 3.1 & -2.3 & 7.2 & 3.3 & 11.7 \\
\hline & 18.0 & 3.3 & 5.5 & 4.6 & 5.4 & 2.4 & 6.4 & 6.5 & 3.0 \\
\hline \multirow[t]{2}{*}{ icariside II } & 3.0 & 4.3 & 8.0 & 11 & 6.4 & 0 & 13.4 & 2.3 & 9.5 \\
\hline & 18.0 & 0.6 & 4.2 & -8.5 & 5.2 & -6.3 & 4.9 & 5.7 & 2.5 \\
\hline \multirow[t]{2}{*}{ icaritin } & 3.0 & 12.3 & 3.6 & 4.3 & 11.2 & 14.3 & 1.8 & -10 & 6.3 \\
\hline & 18.0 & 1.7 & 6.1 & -4.1 & 2.5 & -5.7 & 7.9 & 0.2 & 0.7 \\
\hline
\end{tabular}

Table 4: Stability results of icariin, icariside I, icariside II and icaritin in rabbit serum $(n=3)$. 
Citation: Hu L, Zheng Y, Zhang Y, Qin Z, Dai Y, et al. (2017) Pharmacokinetic Investigation of Main Bioactive Components from Epimedium-Derived Flavonoids in Rabbit Serum by Ultra-Performance Liquid Chromatography Tandem Mass Spectrometry. Med Chem (Los Angeles) 7: 869874. doi: 10.4172/2161-0444.1000444

extraction at ambient temperature for $24 \mathrm{~h}$ and under storage for $8 \mathrm{~h}$ at ambient temperature.

\section{Pharmacokinetic application}

The validated method was successfully applied to investigate the pharmacokinetic of icariside II, the main bioactive component in rabbit serum after oral administration of EF with a single dose of $80 \mathrm{mg} / \mathrm{kg}$. As the main absorbed bioactive component, icariside II could be determined accurately in dosed-rabbit serum. However, the other three xenobiotics (icariin, icariside I and icaritin) appeared in dosed-rabbit serum with concentrations lower than the LLOQ and could not be accurately determined except individual time points. But we still estimate the concentration of icariin, icariside I and icaritin preliminarily by data extrapolation of calibration curves, which could help investigate the dynamic concentration profiles in rabbit serum.

The mean concentration-time profiles of icariin, icariside I, icariside II and icaritin were shown in Figure 3 after oral administration of EF with a single dose of $80 \mathrm{mg} / \mathrm{kg}$. The pharmacokinetic parameters of icariside II were summarized in Table 5. The $\mathrm{T}_{\max }$ and $\mathrm{t}_{1 / 2}$ values were $4.0 \pm 0.0 \mathrm{~h}$ and $1.03 \pm 0.076 \mathrm{~h}$, respectively. The $\mathrm{C}_{\max }$ value was $20.83 \pm$ $3.96 \mathrm{ng} / \mathrm{mL}$. In addition, $\mathrm{AUC}_{0 \text { - }}$ and $\mathrm{AUC}_{0-\infty}$ values were $83.92 \pm 5.34$ and $87.08 \pm 5.17 \mathrm{ng} \cdot \mathrm{h} / \mathrm{mL}$, respectively. $\mathrm{MRT}_{0-\mathrm{t}}$ and $\mathrm{MRT}_{0-\infty}$ values were $4.28 \pm 0.27$ and $4.47 \pm 0.25 \mathrm{~h}$. These results indicate that the main bioactive metabolite, icariside II was more easily absorbed from the EF, while the pharmacokinetic parameters were not available for icariin, icariside I and icariside II. Previous study have indicated that poor oral bioavailability and first-pass effect of prenylflavonoids had been performed by human intestinal Caco- 2 and perfused rat intestinal

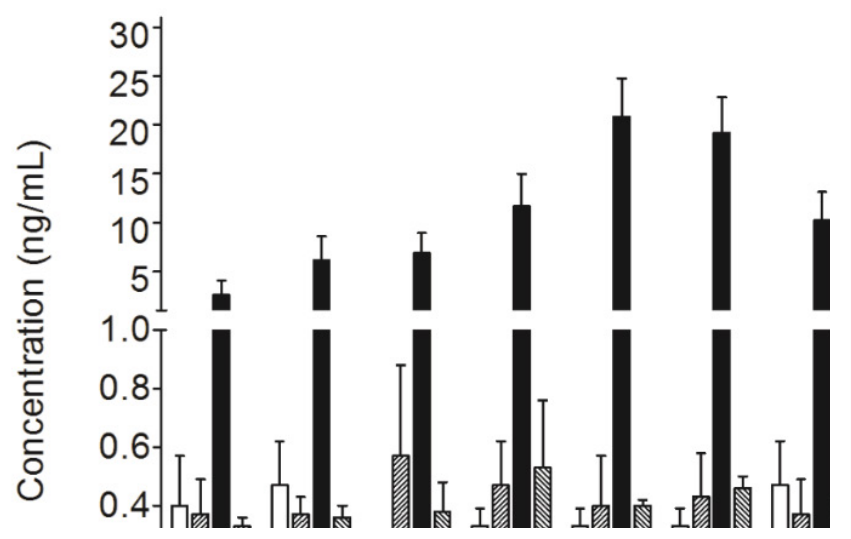

Figure 3: Mean concentration-time profiles of icariin, icariside I, icariside II and icaritin in rabbit serum after oral administration of Epimedium-derived flavonoids extracts.

\begin{tabular}{|c|c|}
\hline Parameters & Values \\
\hline $\mathrm{T}_{\max }(\mathrm{h})$ & $4.0 \pm 0.0$ \\
\hline $\mathrm{C}_{\max }(\mathrm{ng} / \mathrm{mL})$ & $20.83 \pm 3.96$ \\
\hline $\mathrm{t}_{1 / 2}(\mathrm{~h})$ & $1.03 \pm 0.076$ \\
\hline $\mathrm{AUC}_{0-\mathrm{t}}(\mathrm{ng} \mathrm{h} / \mathrm{mL})$ & $83.92 \pm 5.34$ \\
\hline $\mathrm{AUC}_{0-\infty}(\mathrm{ng} \mathrm{h} / \mathrm{mL})$ & $87.08 \pm 5.17$ \\
\hline $\mathrm{MRT}_{0-\mathrm{t}}(\mathrm{h})$ & $4.28 \pm 0.27$ \\
\hline $\mathrm{MRT}_{0-\infty}(\mathrm{h})$ & $4.47 \pm 0.25$ \\
\hline
\end{tabular}

Table 5: Pharmacokinetic parameters of icariside II in rabbit serum after oral administration of Epimedium-derived flavonoids extracts. models [14]. Generally, the in vivo metabolism of Herba Epimedii and its prenylflavonoids could easily be metabolized in gastrointestinal tract following deglycosylation reaction. In this study, EF contain many prenylflavonoids, such as icariin $(\geq 83 \%)$, epimedoside $\mathrm{A}(\leq 1.65 \%)$, hexandraside $\mathrm{F}(<0.91 \%)$, epimedin $\mathrm{A}(<1.18 \%)$, epimedin $\mathrm{B}(\leq 1.52 \%)$, epimedin $\mathrm{C}(<4.1 \%)$ and icariside II $(<1.84 \%)$. Icariside II have proved be one of the major intermediate decomposition products of these prenylflavonoids [23]. This may explain the higher absorption of $\mathrm{C}_{\max }$ observed for icariside II in the present study compared to previously published reports [24].

In conclusion, a rapid and sensitive UPLC-Q-TOF/MS method has been established for simultaneous analysis of icariin, icarisid I, icarisid II and icaritin in rabbit serum. Furthermore, the validated method was successfully applied for the pharmacokinetics of EF in rabbit serum. This study can enlarge our knowledge of in vivo metabolic fate of $\mathrm{EF}$ after oral administration. Furthermore, the bioactivity evaluation would be contributed to clarifying how the EF reduce the risk of Steroid-induced Osteonecrosis in rabbit.

\section{Acknowledgements}

This work was supported by National Natural Science Foundation of ChinaResearch Grant Council of Hong Kong (Grant No. 30831160510).

\section{References}

1. Editorial Committee of Pharmacopoeia of Ministry of Health PR China (2015) The Pharmacopeoia of People's Republic of China (Part 1), China, Chemical Industry Press, Beijing, p: 327.

2. Xu F, Ding Y, Guo Y, Liu B, Kou Z, et al. (2016) Anti-osteoporosis effect of Epimedium via an estrogen-like mechanism based on a system-level approach. J Ethnopharmacol 177: 148-160.

3. Ma H, He X, Yang Y, Li M, Hao D, et al. (2011) The genus Epimedium: An ethnopharmacological and phytochemical review. J Ethnopharmacol 134 519-541.

4. Peng S, Zhang G, He Y, Wang X, Leung P, et al. (2009) Epimedium-derived flavonoids promote osteoblastogenesis and suppress adipogenesis in bone marrow stromal cells while exerting an anabolic effect on osteoporotic bone. Bone 45: 534-544.

5. Zhang G, Wang XL, Sheng H, Xie X, He Y, et al. (2009) Constitutional flavonoids derived from Epimedium dose-dependently reduce incidence of steroidassociated osteonecrosis not via direct action by themselves on potential cellular targets. Plos ONE 4: e6419.

6. Zhang G, Qin L, Sheng H, Yeung K, Yeung H, et al. (2007) Epimediumderived phytoestrogen exert beneficial effect on preventing steroid-associated osteonecrosis in rabbits with inhibition of both thrombosis and lipid-deposition. Bone 40: 685-692.

7. Zhang G, Qin L, Shi Y (2007) Epimedium-Derived Phytoestrogen Flavonoids Exert Beneficial Effecton Preventing Bone Loss in Late Postmenopausal Women: A 24-Month Randomized, Double-Blind and Placebo-Controlled Trial. J Bone Miner Res 22: 1072-1079.

8. Sheng H, Zhang G, Qin L, Wang Y, Wang X, et al. (2008) Herbal Epimediumderived bioactive compound icariin reduces the incidence of steroid-associated osteonecrosis. Bone 43: S94-S110.

9. Zhang G, Qin L, Sheng H, Wang X, Wang Y, et al. (2009)A novel semisynthesized small molecule icaritin reduces incidence of steroid-associated osteonecrosis with inhibition of both thrombosis and lipid-deposition in a dose-dependent manner. Bone 44: 345-356.

10. Qin L, Yao D, Zheng L, Liu W, Liu Z, et al. (2015) Phytomolecule icaritin incorporated PLGA/TCP scaffold for steroidassociated osteonecrosis: Proofof-concept for prevention of hip joint collapse in bipedal emus and mechanistic study in quadrupedal rabbits. Biomaterials 59: 125-143.

11. Chen SH, Lei M, Xie XH Zheng L, Yao D, et al. (2013) PLGA/TCP composite scaffold incorporating bioactive phytomolecule icaritin for enhancement of bone defect repair in rabbits. Acta Biomater 9: 6711-6722.

12. Peng S, Zhang G, Zhang BT, Guo B, He Y, et al. (2013) The beneficial effect of 
Citation: Hu L, Zheng Y, Zhang Y, Qin Z, Dai Y, et al. (2017) Pharmacokinetic Investigation of Main Bioactive Components from Epimedium-Derived Flavonoids in Rabbit Serum by Ultra-Performance Liquid Chromatography Tandem Mass Spectrometry. Med Chem (Los Angeles) 7: 869874. doi: 10.4172/2161-0444.1000444

icaritin on osteoporotic bone is dependent on the treatment initiation timing in adult ovariectomized rats. Bone 55: 230-240.

13. Xie X (2010) R \& D of an Innovative Composite Scaffold Incorporated with Phytoestrogenic Icaritin for Treatment of Steroid-associated Osteonecrosis Lesion in Rabbits, p: 193

14. Chen Y, Zhao YH, Jia XB, Hu M (2008) Intestinal absorption mechanisms of prenylated flavonoids present in the heat-processed Epimedium koreanum Nakai (Yin Yanghuo). Pharm Res-dordr 25: 2190-2199.

15. Zhou J, Ma YH, Zhou Z, Chen Y, Wang Y, et al. (2015) Intestinal Absorption and Metabolism of Epimedium Flavonoids in Osteoporosis Rats. Drug Metab Dispos 43: 1590-1600.

16. Liu M, Yao Z, Zhang Y, Dai Y, Qin Z, et al. (2011) Impact of Two Pretreatment Methods on the Matrix Effect of Epimedium-derived Flavonoid Compounds in Rabbit Serum by LC-MS/MS. J Instrumental Anal 30: 1006-1012.

17. Yao Z, Liu M, Dai Y, Zhang Y, Qin Z, et al. (2011) Metabolism of Epimediumderived Flavonoid Glycosides in Intestinal Flora of Rabbits and Its Inhibition by Gluconolactone. Chin J Nat Med 9: 461-465.

18. Zhao H, Fan M, Fan L, Sun J, Guo D (2010) Liquid chromatography-tandem mass spectrometry analysis of metabolites in rats after administration of prenylflavonoids from Epimediums. J Chromatogr B Analyt Technol Biomed Life Sci 878: 1113-1124.

19. Qin Z, Dai Y, Yao Z, He L, Wang Q, et al. (2016) Study on chemical profiles and metabolites of Allii Macrostemonis Bulbus as well as its representative steroidal saponins in rats by ultra-performance liquid chromatography coupled with quadrupole time-of-flight tandem mass spectrometry. Food Chemistry 192 499-515.

20. Qin Z, Lin P, Dai Y, Yao Z, Wang L, et al. (2016) Quantification and semiquantification of multiple representative components for the holistic quality control of Allii Macrostemonis Bulbus by ultra high performance liquid chromatography with quadrupole time-of-flight tandem mass spectrometry. $J$ Sep Sci 39: 1834-1841.

21. US FDA (2001) Guidance for Industry: Bioanalytical Method Validation, US Department of Health and Human Services, Center for Drug Evaluation and Research (CDER)

22. Matuszewski BK, Constanzer ML, Chavez-Eng CM (1998) Matrix Effect in Quantitative LCMSMS Analyses of Biological Fluids: A Method for Determination of Finasteride in Human Plasma at Picogram Per Milliliter Concentrations. Anal Chem 70: 882-889.

23. Zhao H, Fan M, Fan L, Sun J, Guo D (2010) Liquid chromatography-tandem mass spectrometry analysis of metabolites in rats after administration of prenylflavonoids from Epimedium. J Chromatogr B Anal Technol Biomed Life Sci 878: 1113-1124.

24. Xu W, Zhang Y, Yang M, Shen Z, Zhang X, et al. (2007) LC-MS/MS method for the simultaneous determination of icariin and its major metabolites in rat plasma. J Pharm Biomed Anal 45: 667-672. 